\title{
The influence of proteasome inhibitor on the expression of cardiomyocytes damage markers after incubation with doxorubicin
}

\author{
Sylwia Tereszkiewicz ${ }^{1}$, Wlodzimierz Matysiak ${ }^{2}$, Slawomir MandziUk ${ }^{3}$, \\ Agnieszka Korga ${ }^{1}$, Malgorzata Oleksiejuk ${ }^{4}$, Marcin Hejna ${ }^{5}$, \\ Agnieszka Korobowicz-Markiewicz ${ }^{6}$, Magdalena Iwan ${ }^{1}$, Jaroslaw Dudka ${ }^{1}$
}

\author{
${ }^{1}$ Independent Medical Biology Unit, Medical University of Lublin, Poland \\ ${ }^{2}$ Department of Histology and Embryology, Medical University of Lublin, Poland \\ ${ }^{3}$ Department of Pneumology Oncology and Allergology, Medical University of Lublin, Poland \\ ${ }^{4}$ IMMUNIQ, Beata Solon-Gogol, Sasiedzka 1, 44-240 Zory, Poland \\ ${ }^{5}$ Regional Dental Clinic Independent Public Health Care, Lubartowska 58, Lublin, Poland \\ ${ }^{6}$ Department of Pediatric Pulmonology and Rheumatology, Medical University of Lublin, Poland
}

\begin{tabular}{ll}
\hline ARTICLE INFO & ABSTRACT \\
\cline { 1 - 2 } $\begin{array}{l}\text { Received 11 June 2014 } \\
\text { Accepted 13 June 2014 }\end{array}$ & $\begin{array}{l}\text { The aim of the study was to verify the thesis that the cardiotoxic effects of doxorubicin } \\
\text { are connected with activation of the ubiquitin - proteasome pathway followed by protein } \\
\text { degradation. The expression of myocardial damage markers - fatty acid binding protein } \\
\text { (H-FABP) and brain natriuretic peptide (BNP) was evaluated in rat fetal cardiomyocytes } \\
\text { Keywords: } \\
\text { doxorubicin, } \\
\text { BNP, }\end{array}$ \\
$\begin{array}{l}\text { H-FABP, } \\
\text { proteasom. }\end{array}$ & $\begin{array}{l}\text { level of H-FABP and BNP protein under the influence of doxorubicin was decreased below } \\
\text { the detection threshold with unchanged (H-FABP) or elevated (BNP) mRNA expression } \\
\text { level. Against the expectations, the inhibitor of proteasome did not abolish this effect. The } \\
\text { observed abnormal expression of BNP and H-FABP protein after doxorubicin treatment } \\
\text { makes their diagnostic significance in anthracycline cardiotoxicity questionable. }\end{array}$ \\
\hline
\end{tabular}

\section{INTRODUCTION}

Doxorubicin has been used in cancer therapy for more than 40 years. In addition to the antitumor effect, it also has clinically significant toxic effects against myocardium [5]. Previous studies have shown that the mechanism of the antitumor effect is different from the mechanism of cardiotoxicity. The antitumor effect of doxorubicin is primarily due to the inhibition of DNA and RNA synthesis by forming covalent bonds with nucleic acids, as well as an interaction with helicase and topoisomerase II [18]. It was also shown that doxorubicin acts on cancer cells via the ubiquitin-proteasome pathway as its inhibitor [12]. However, toxic effects on the myocardium are associated with the generation of oxidative stress, which leads to the damage of proteins, lipids, and DNA [19]. It was also shown that doxorubicin in cardiomyocytes can act as an activator of the proteasome. Intensifying the properties of the proteolytic

Corresponding author

e-mail: agnieszka.korga@umlub.pl ubiquitin-proteasome pathway, the drug leads to degradation of proteins and critical cardiac transcription regulators which are important for cardiac functioning [10]. It was shown that doxorubicin can cause a decrease in cardiac proteins such as fatty acid binding protein (H-FABP) and brain natriuretic peptide (BNP) - potential markers of anthracycline cardiotoxicity, which calls into question the validity of its evaluation in patients treated with doxorubicin $[9,17]$. There was no evidence, however, whether abnormal expression of these proteins is associated with the activation of the ubiquitinproteasome pathway. Therefore, the aim of this study was to evaluate the expression of H-FABP and BNP at the level of protein and mRNA in rat fetal cardiomyocytes treated with doxorubicin and the proteasome inhibitor - bortezomib.

\section{METHODS}

\section{Cell culture}

Tests were carried out on cell line H9C2. These are cells that are genetically subclone of the original cell line derived from a rat embryo heart tissue BD1X. The culture was grown at $37^{\circ} \mathrm{C}$ in a $5 \% \mathrm{CO}_{2}$ atmosphere. 
Cells were treated with bortezomib (LClabs, USA) which was added to the cultures for 30 minutes prior to administration of doxorubicin (EbewePharma, Austria). The culture was terminated after 24 hours' incubation with doxorubicin, and protein and mRNA were isolated.

Doses of the compounds and the incubation time were selected basing on the results of the MTT cytotoxicity assay and the evaluation of cell morphology with a microscope.

Table 1. Doses of doxorubicin and bortezomib used in the study

\begin{tabular}{|l|c|c|}
\hline & Doxorubicin & Bortezomib \\
\hline Control & - & - \\
\hline B1 & - & $1 \mu \mathrm{M}$ \\
\hline B100 & - & $100 \mathrm{nM}$ \\
\hline A1 & $1 \mu \mathrm{M}$ & - \\
\hline A5 & $5 \mu \mathrm{M}$ & - \\
\hline B1 A1 & $1 \mu \mathrm{M}$ & $1 \mu \mathrm{M}$ \\
\hline B1 A5 & $5 \mu \mathrm{M}$ & $1 \mu \mathrm{M}$ \\
\hline B100 A1 & $1 \mu \mathrm{M}$ & $100 \mathrm{nM}$ \\
\hline B100 A5 & $5 \mu \mathrm{M}$ & $100 \mathrm{nM}$ \\
\hline
\end{tabular}

\section{Determination of cytotoxicity of test compounds using the MTT assay}

Cells were seeded in culture plates 12 -well, after about 20000 cells per well and incubated with test compounds (at the above concentrations). Then, after 4, 24 and 72 hours $200 \mathrm{ml}$ of solution dye was added per $1 \mathrm{ml}$ of culture medium at the concentration of $4 \mathrm{mg} / \mathrm{ml}$. After 4 hours, the MTT solution was pulled down and $1 \mathrm{ml}$ of DMSO was added. The absorbance was measured using a plate reader at a wavelength of $\lambda=540 \mathrm{~nm}$.

\section{RNA Isolation}

RNA was isolated using TRI reagent (Sigma-Aldrich, USA) according to the manufacturer's instructions. The integrity of the RNA was checked using electrophoresis in $1.5 \%$ agarose gel. The concentration and purity of RNA were assessed by measuring the absorbance at a wavelength of $\lambda$ $=260$ and $\lambda=280$.

\section{Reverse transcription}

Reverse transcription was performed using High Capacity cDNA Reverse Transcription Kit (Life Technologies, USA) according to the manufacturer's instructions.

\section{Determination of gene expression by real - time PCR}

The relative expression of genes BNP and H-FABP was measured by reaction Real-time PCR, $\Delta \Delta \mathrm{Ct}$ method, using $\beta$-actin as an endogenous control.

The reaction was carried out in AppliedBiosystems 7500 Fast, using Fast Universal Master Mix and primer sets and probes: TaqMan Gene Expression Assays Rn 00577366 m1Fabp3, Rn00580641_m1Nppb, Rn00667869_m1Actb.

\section{Determination of BNP and H-FABP protein expression} by Western Blot

To isolate proteins cardiomyocytes were treated with lysis buffer and cell lysates were centrifuged at $12000 \mathrm{rpm}$ for $20 \mathrm{~min}$. The protein concentration in supernatants was determined spectrophotometrically by the Bradford method.

Proteins from cell lysates were separated in denaturing conditions on a polyacrylamide gel at the concentration of $15 \%$, at $120 \mathrm{~V}$ for approximately 2 hours. Transfer of proteins was then carried out from the gel to a nitrocellulose membrane at a constant voltage of $30 \mathrm{~V}$ for $1.5 \mathrm{hr}$.
To visualize the proteins the appropriate antibodies were used (RbpAb a cardiac FABP, ab 102075,RbpAb a pro BNP, ab 32842, RbpAb a beta Actinab 8227, Abcam, United Kingdom), with a set of reagents Western Breeze Chromogenic Western Blot Immunodetection Kit (Invitrogen, USA), while $\beta$-actin was used as a loading control. The intensity of the resulting bands was compared by densitometry using 1D Imane Analysis Software (Kodak, USA).

\section{RESULTS}

There were only slight changes in mRNA expression for H-FABP gene (Fig. 1) while the expression of BNP mRNA increased in all tested cultures, especially after bortezomib treatment (Fig. 2)

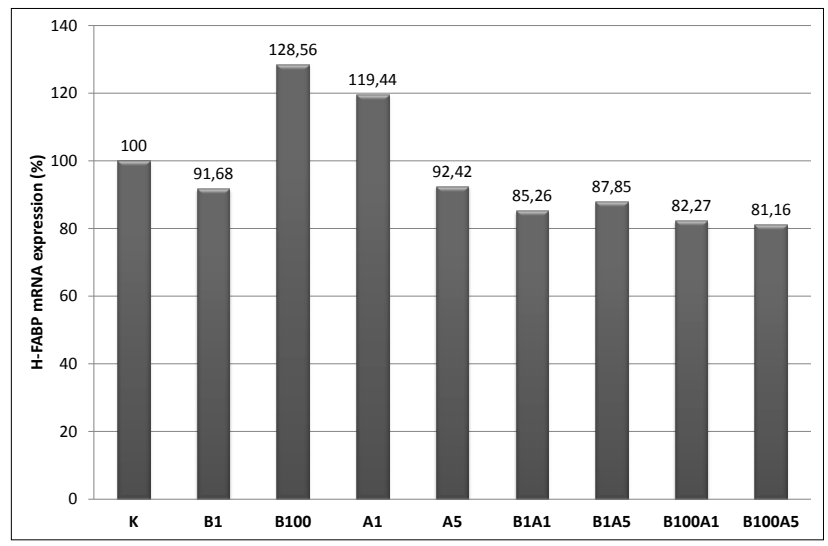

Figure 1. The level of H-FABP mRNA expression, expressed as \% of expression in control cells

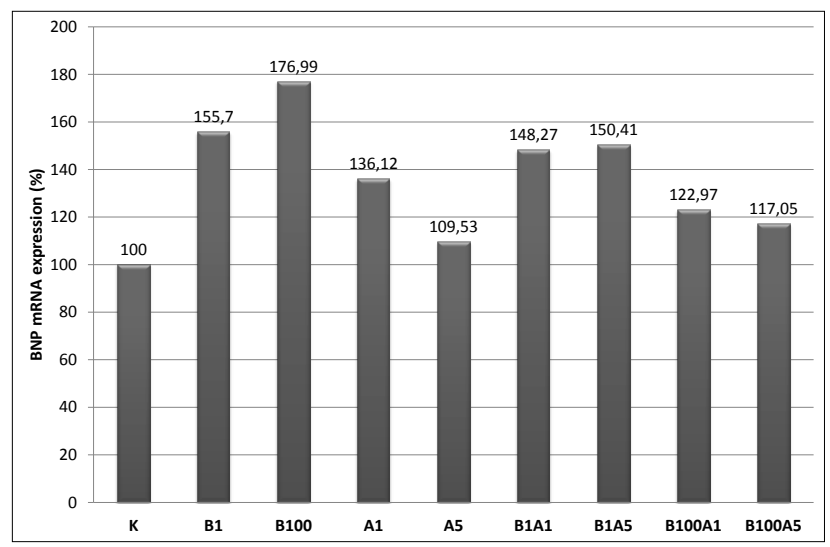

Figure 2. The level of BNP mRNA expression, expressed as \% of expression in control cells

Expression of H-FABP protein after administration of bortezomib falls below the control. It was the lowest in cells treated with a higher dose of the drug. In other assays protein was undetectable by Western blot (Fig. 3).

BNP protein expression was the highest after incubation with bortezomib at the concentration of 1 micromol. However, the protein level was lower after the administration of bortezomib at the concentration of $100 \mathrm{nmol}$. In the remaining samples, protein expression was undetectable with the applied method (Fig. 4). 


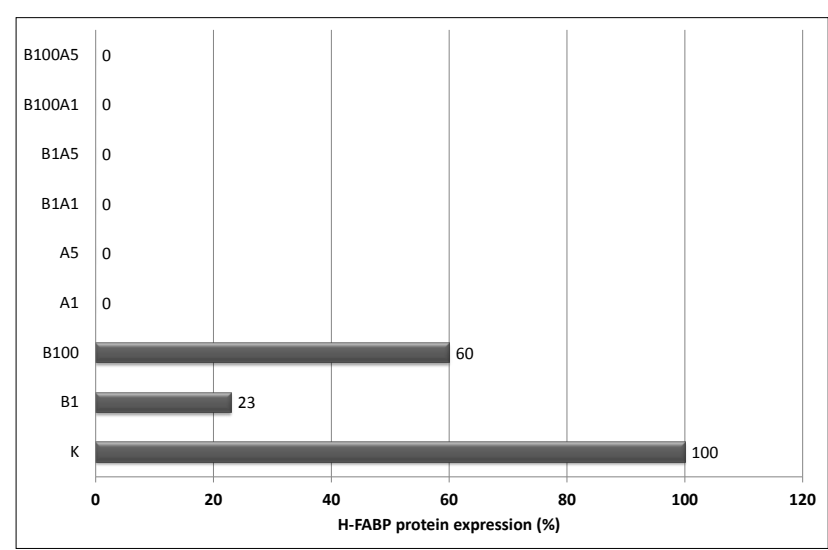

Figure 3. The level of H-FABP protein expression, expressed as \% of expression in control cells

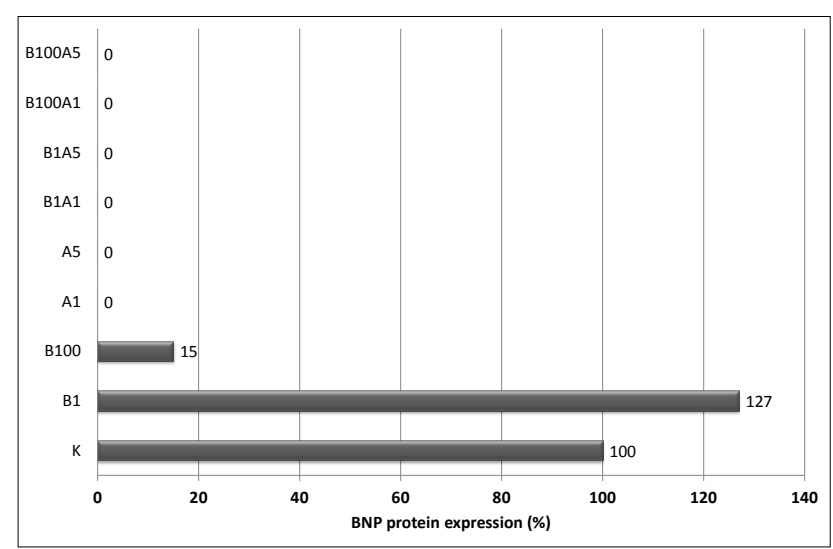

Figure 4. The level of BNP protein expression, expressed as \% of expression in control cells

\section{DISCUSSION}

Doxorubicin is toxic to the heart muscle mainly through the induction of oxidative stress. In addition, it disrupts homeostasis of calcium and iron [5] and acts as an activator of the ubiquitin - proteasome pathway in myocardial cells $[10,14]$. It has been sought factors that allow prevent anthracycline cardiotoxicity as well as diagnostic markers by which early changes in the heart of patients treated with doxorubicin can be detected.

There are contrary result of studies on the usefulness of H-FABP as a marker of anthracycline cardiotoxicity. Chronic administration of doxorubicin causes a decrease in the expression of mRNA for H-FABP. The decrease of expression was shown to be dose-dependent. Also, it is secondary to its cytotoxic activity by inhibiting synthesis of DNA, RNA and protein [15]. Inhibition of H-FABP mRNA expression also occurs through the production of reactive oxygen species [16]

In our study, there were no changes in mRNA expression for H-FABP. Bortezomib alone in a lower dose resulted in a $30 \%$ increase, while in the cells treated with the two compounds there were observed small (less than 20\%) decreases in the expression of this gene. Different changes were observed at the protein level - H-FABP expression was seen only in the control cardiomyocytes and in cells that were treated with two concentrations of bortezomib, while expression in the cells cultured in the presence of doxorubicin, doxorubicin and bortezomib was undetectable. This means that the synthesis of H-FABP was not inhibited at the stage of transcription, but rather on the subsequent stages. We cannot determine whether the observed phenomenon resulted from increased proteasome activation and degradation of the protein of interest as bortezomib did not remove the effect caused by doxorubicin.

Bortezomib is an anticancer drug, but it is also characterized by high toxicity to normal cells. Bortezomib acts on tumor cells by way of inhibiting the ubiquitin - proteasome [1] as well as by inhibiting the activation of NF-kappaB [8]. In the literature there have been reports on toxic effects of bortezomib whereby bortezomib by inhibiting the ubiquitin - proteasome leads to cell growth inhibition in the myocardium, which is induced by NF-kappaB. It was found that bortezomib inhibiting the ubiquitin - proteasome leads to block translation initiation process, which contributes to achieving balance between the degradation and the synthesis of proteins [8]. It is likely, therefore, that in the experiment the concentration of bortezomib was too small to abolish the effect induced by doxorubicin or it inhibits expression of the protein at the stage of translation.

BNP protein belonging to the family of natriuretic hormones [6] is released as a result of excessive stretching, especially cardiac left ventricle, and reflects hypoxia and ventricular wall stress [17]. In the experiment there was observed only a slight increase in BNP mRNA expression in cardiomyocytes after activation with doxorubicin at lower levels. The increase was also found after treatment with bortezomib alone and in cardiomyocytes cultured in the presence of both compounds. Bortezomib results obtained are consistent with studies that have been performed on patients who demonstrated that treatment with bortezomib increases the BNP precursor of NT-proBNP [20]. Other studies have shown that after the administration of bortezomib an increase in the concentration of BNP is observed. BNP concentration falls then simultaneously with the cessation of bortezomib [7]

However, no BNP gene expression inhibition by doxorubicin was observed by other researchers [4]. The results are also contrary to reports according to which doxorubicin toxic effect on cardiomyocytes leads to increased protein expression of BNP and BNP correlates with the dose of doxorubicin [13]. At the protein level (Western Blot), as in the case of H-FABP, expression was found only in cardiomyocytes of control and those treated with bortezomib. In other cultures BNP protein level was below the detection threshold. No BNP expression after incubation with doxorubicin in the experiment can be applied to the results of Aichara et al [2]. They showed that after the administration of doxorubicin the reduced level of BNP is caused by the action of hydrogen peroxide. Decrease in the expression of BNP precursor proBNP observed in studies conducted by Betheya et al [3]. In patients treated with doxorubicin, there were reductions in the left ventricular ejection fraction of myocardial infarction.

Protein levels of both H-FABP and BNP decreased significantly below control. Only bortezomib at $1 \mathrm{mM}$ did not change significantly the expression of the protein. In the case of doxorubicin and doxorubicin in combination with 
bortezomib the the expression was below the detection threshold. Given the fact that the level of mRNA for these molecules was above the control or not changed, and the expression of the protein decreased or was not detectable it can be assumed that there is abnormal protein synthesis due to disturbances on the posttranscriptional stage. The results cast doubt on the desirability of determining protein H-FABP and BNP as markers of the use of doxorubicin cardiotoxicity.

The aim of the experiment was to verify the thesis that the cardiotoxicity of doxorubicin is connected with protein degradation due to proteasom activation and bortezomib the proteasome inhibitor abolishes this effect. The results, however, did not confirm this assumption. On the basis of the study it can be concluded that the observed decrease in the level of proteins was due to doxorubicin inducing impacts on the process of translation. The supposition is confirmed by the fact that the increase in mRNA expression in cardiomyocytes does not translate into increased levels of protein. Although the studies have shown that bortezomib has the ability to decrease cardiotoxicity the results showed that the mechanism for it is unclear.

\section{ACKNOWLEDGEMENT}

The paper was developed using the equipment purchased within the Project „The equipment of innovative laboratories doing research on new medicines used in the therapy of civilization and neoplastic diseases" within the Operational Program Development of Eastern Poland 2007-2013, Priority Axis I Modern Economy, Operations I.3 Innovation Promotion.

\section{REFERENCES}

1. Adams J.: Development of the Proteasome Inhibitor PS-341. The Oncologist, 7, 9, 2002.

2. Aihara Y. et al.: Doxorubicin Represses CARP Gene Transcription Through the Generation of Oxidative Stress in Neonatal Rat Cardiac Myocytes: Possible Role of Serine/Threonine Kinase - dependent Pathways. J. Mol. Cell Cardiol., 32, 1401, 2000.

3. Batheja R. et al.: Paradoxical decrease in plasma N-terminal-proBNP (NTproBNP) levels in patients receiving doxorubicin chemotherapy and declining left ventricular ejection fraction. J. Am. Coll. Cardiol., 47, 66A, 2006.

4. Chen S., Garami M., Gardner D. G.: Doxorubicin Selectively Inhibits Brain Versus Atrial Natriuretic Peptide Gene Expression in Cultured Neonatal Rat Myocytes. Hypertension, 34, 1223, 1999.

5. Dudka J.: The role of reactive oxygen and nitrogen species in calcium and iron homeostasis dysregulation in anthracycline cardiotoxicity. Postepy Hig. Med. Dosw, 60, 241, 2006.

6. Dudka J. et al.: The diagnosis of anthracycline-induced cardiac damage and heart failure. Postepy Hig. Med. Dosw , 63, 225, 2009.

7. Hacihanefioglu A., Tarkun P., GonulluE.: Acute severe cardiac failure in a myeloma patient due to proteasome inhibitor bortezomib. Int. J. Hematol., 88, 219, 2008.

8. Hedhli N., Depre Ch.: Proteasome inhibitors and cardiac cell growth. Cardiovasc. Res., 85, 321, 2010.

9. Korga A. et al.: The redox imbalance and the reduction of contractile protein content in rat hearts administered with L-thyroxine and Doxorubicin. Oxid. Med. Cell Longev., 681367, 2012.

10. Liu J. et al.: A therapeutic dose of doxorubicin activates ubiquitinproteasome system-mediated proteolysis by acting on both the ubiquitination apparatus and proteasome. Am. J. Physiol. Heart Circ. Physiol., 295, H2541, 2008.

11. Lyu Y. L. et al.: TopoisomeraseIIb-Mediated DNA Double-Strand Breaks: Implications in Doxorubicin Cardiotoxity and Prevention by Dexrazoxane. Cancer Res., 67, 8839, 2007.
12. Minotti G. et al.: Anthracyclines: Molecular Advances and Pharmacologic Developments in Antitumor Activity and Cardiotoxity. Pharmacol. Rev., 56, 185, 2004.

13. Pichon M. F. et al.: Drug - induced Cardiotoxity Studied by Longitudinal B-Type Natriuretic Peptide Asays and Radionuclide Ventryculography. In Vivo, 19, 567, 2005.

14. Ranek M. J., Wang X.: Activation of the Ubiquitin Proteasome System in Doxorubicin Cardiomyopathy. Curr. Hypertens. Rep., 11, 389, 2009.

15. Sayed-Ahmad M. M. et al.: Protection by L-Carnitine Against the Inhibition of Gene Expression of Heart Fatty Acid Binding Protein by Chronic Administration of Doxorubicin. Journal of the Egyptian Nat. Cancer Inst., 4, 275, 2000.

16. Sayed-Ahmed M. M. et al.: Inhibition of gene expression of heart fatty acid binding protein and organic cation/carnitine transporter in doxorubicin cardiomyopathic rat model. Eur. J. Pharmacol., 640, 143, 2010.

17. Sun Y. et al.: B-Type Natriuretic Peptide-Induced Cardioprotection against Reperfusion Is Associated with Attenaution of Mitochondrial Permeability Transition. Biol. Pharm. Bull, 32, 1545, 2009.

18. Szuławska A., Czyż M.: Molecular mechanisms of anthracyclines action. Postepy Hig. Med. Dosw., 60, 78, 2006.

19. Tsutsui H., Kinugawa S., Matsushima S.: Oxidative Stress and Mitochondrial DNA Damage in Heart Failure",Circ. J., Suppl Am, A-31, 2008.

20. Voortman J., GiacconeG.: Severe reversible cardiac failure after bortezomib treatment combined with chemotherapy in a non-small cell lung cancer patient: a case report. BMC Cancer, 6, 129, 2006. 